\title{
FAR ULTRAVIOLET SPECTROSCOPIC EXPLORER OBSERVATIONS OF THE SUPERNOVA REMNANT N49 IN THE LARGE MAGELLANIC CLOUD ${ }^{1}$
}

\author{
William P. Blair ${ }^{1}$, Ravi Sankrit ${ }^{1}$, Robin Shelton ${ }^{1}$, Kenneth R. Sembach ${ }^{1}$, H. Warren \\ Moos $^{1}$, John C. Raymond ${ }^{2}$, Donald G. York ${ }^{3}$, Paul D. Feldman ${ }^{1}$, Pierre Chayer ${ }^{1}$, \\ Edward M. Murphy ${ }^{1}$, David J. Sahnow ${ }^{1}$, And Erik Wilkinson ${ }^{4}$ \\ Draft version May 6, 2000
}

\begin{abstract}
We report a Far Ultraviolet Spectroscopic Explorer satellite observation of the supernova remnant N49 in the Large Magellanic Cloud, covering the $905-1187 \AA$ spectral region. A 30" square aperture was used, resulting in a velocity resolution of $\sim 100 \mathrm{~km} \mathrm{~s}^{-1}$. The purpose of the observation was to examine several bright emission lines expected from earlier work and to demonstrate diffuse source sensitivity by searching for faint lines never seen previously in extragalactic supernova remnant UV spectra. Both goals were accomplished. Strong emission lines of O VI $\lambda \lambda 1031.9,1037.6$ and C III $\lambda 977.0$ were seen, Doppler broadened to $\pm 225 \mathrm{~km} \mathrm{~s}^{-1}$ and with centroids red-shifted to $350 \mathrm{~km} \mathrm{~s}^{-1}$, consistent with the LMC. Superimposed on the emission lines are absorptions by C III and O VI $\lambda 1031.9$ at $+260 \mathrm{~km} \mathrm{~s}^{-1}$, which are attributed to warm and hot gas (respectively) in the LMC. The O VI $\lambda 1037.6$ line is more severely affected by overlying interstellar and $\mathrm{H}_{2}$ absorption from both the LMC and our galaxy. N III $\lambda 989.8$ is not seen, but models indicate overlying absorption severely attenuates this line. A number of faint lines from hot gas have also been detected, many of which have never been seen in an extragalactic supernova remnant spectrum.
\end{abstract} ISM

Subject headings: ISM: individual (N49) — Magellanic Clouds — shock waves — supernova remnants — ultraviolet:

\section{INTRODUCTION}

Optical/UV spectra of supernova remnants (SNRs) contain emission lines from a broad range of ionization and excitation states of many intermediate mass elements. Observations with the Voyager Ultraviolet Spectrometers (Blair et al. 1991a; Vancura et al. 1993, Blair et al. 1995a) and the Hopkins Ultraviolet Telescope (HUT; Blair et al. 1991b; Long et al. 1992, Blair et al. 1995b) have revealed the stronger lines expected in this spectral region. However, the low spectral resolution of these earlier observations, contamination by residual airglow emission lines, and concerns about overlying absorption have compromised the utility of these data.

A case in point is the bright X-ray and optical SNR known as N49 in the Large Magellanic Cloud (LMC; see Mathewson et al. 1983; Long et al. 1981). This object was observed with HUT (Vancura et al. 1992b), but the residual airglow in the sub-Ly $\alpha$ region caused considerable difficulty in measuring the intrinsic SNR emissions. Vancura et al. (1992b) were able to infer the presence and approximate total strength of the O VI $\lambda \lambda 1031.9,1037.6$ doublet, but could not separate the lines clearly from the surrounding airglow.

In this paper, we report Far Ultraviolet Spectroscopic Explorer satellite (FUSE) observations of N49. These observations resolve airglow emission from intrinsic SNR emission lines, and allow us to investigate intrinsic line profiles and overlying absorptions. We also report a number of fainter emission lines expected from comparison with shock model calculations or coronal emissions from hot gas. These observations demonstrate the capabilities of FUSE for line profile analysis in SNRs and for detecting faint, diffuse emissions by virtue of its sensitivity and extremely low background levels.

\section{OBSERVATIONS AND REDUCTIONS}

The observations were obtained with the FUSE satellite (Moos et al. 2000, Sahnow et al. 2000) during the in-orbit checkout period. FUSE was pointed toward N49 on 1999 Sept. 21-22 UT, for 34 ks total integration time, about $14 \mathrm{ks}$ of which was during orbital night. We used the $30^{\prime \prime}$ square apertures to maximize throughput. However, the emission from many different filaments, with differing densities, effective reddenings, and shock velocities, are being sampled simultaneously (cf. Vancura et al. 1992a,b). Thus, the FUSE observation only samples the "global" nature of the N49 emissions.

FUSE operates using four telescope channels which are intended to be co-aligned for optimal performance. At the early epoch of these observations, only three channels were approximately aligned. Figure 1 shows the location of the $\mathrm{LiF}$ apertures (solid line, aligned to $3^{\prime \prime}$ ) and the $\mathrm{SiC} 2$ aperture (dashed line) on an [O III] optical image. The $\mathrm{SiC} 2$ channel was offset such that the observed counts in the spectral overlap region are down by a factor of two from what would have been expected based on the relative effective areas of the channels. The SiC1 channel was not aligned, and contained only airglow emissions.

\footnotetext{
${ }^{1}$ Department of Physics and Astronomy, The Johns Hopkins University, 3400 N. Charles Street, Baltimore, MD 21218

${ }^{2}$ Harvard-Smithsonian Center for Astrophysics, 60 Garden St., Cambridge, MA 02138

${ }^{3}$ University of Chicago, 5640 S. Ellis Ave., Chicago, IL 60637

${ }^{4}$ University of Colorado, Campus Box 593, 1255 38th St., Boulder, CO 80302
} 
The data reductions and calibrations are only approximate at this point, consisting of either pre-flight or preliminary in-flight algorithms and calibration values. We work primarily with the raw time-tagged "counts" data sets, and collapse the lines into one-dimensional spectra from each of the three channels that contained useful data. We also applied a preliminary flux calibration to assess approximate relative line intentisites. One can inspect subsets of these data (for instance, the "night only" data) to eliminate transient events or to identify features arising from airglow. Because the resolution of the filled-aperture data is already degraded, we have simply co-added multiple channels whenever a region is covered by more than one.

\section{DISCUSSION}

The FUSE spectrum of N49 demonstrates substantial advantages over previous observations in this spectral region. The SNR emission lines are not only separated from contaminating airglow emission lines, but the kinematics and line profiles of the SNR lines can also be assessed directly. Also, the detection of a number of faint emission lines that had not been detected previously in an extragalactic SNR demonstrates FUSE's excellent sensitivity to faint diffuse emissions (cf. also Shelton et al. 2000). This bodes well for future FUSE observations of nebular emissions that are not as intrinsically complicated as the situation we observe in N49. Table 1 summarizes the lines detected from the SNR and can be used to estimate the observed relative line intensities. Because some regions were observed by multiple channels and the channels were not in perfect alignment, we list measured fluxes from separate channels for O VI.

\subsection{Strong Lines}

In Figure 2a, we show the spectral region from 1020 $1045 \AA$ from the total and night only data sets, a region where all three active channels could be summed. This region contains several airglow emission lines (from $\operatorname{Ly} \beta$ and $\mathrm{O}$ I) and the O VI doublet from the SNR. The airglow lines fill the aperture, and indicate the filled slit spectral resolution, which is $0.3 \AA$ or $\sim 100 \mathrm{~km} \mathrm{~s}^{-1}$. The SNR O VI $\lambda 1031.9$ line is separated from the airglow lines, while some minor contamination of O VI $\lambda 1037.6$ is caused by the O I $\lambda 1039.2$ feature. (This contamination goes away in the night-only data section.) The SNR lines are much wider than the airglow features, broadened by the Doppler motion of material within the aperture. The FWHM of the $1032 \AA$ line is $450 \mathrm{~km} \mathrm{~s}^{-1}$, consistent with narrow slit optical echelle spectra (cf. Vancura et al. 1992a) and a filled slit assumption. Figure $2 \mathrm{~b}$ shows the $970-995 \AA$ spectral region for comparison. (This region was only sampled with a single $\mathrm{SiC}$ channel.) The primary SNR line detected in this region is C III $\lambda 977.0$, although conspicuous by its absence is N III $\lambda$ 989.8. (This will be discussed below.)

In Figure 3 we show these three strong lines on a velocity scale for comparison. Several points are immediately obvious. First of all, the lines are all considerably redshifted from rest velocity. With the airglow lines adjusted to their rest wavelengths, the centroids of the $\mathrm{O}$ VI lines are seen to be shifted by about $+350 \mathrm{~km} \mathrm{~s}^{-1}$. This is higher than the mean LMC velocity but consistent with previous observations of N49 (cf. Shull 1983; Chu \& Kennicutt 1988).
Secondly, the observed profiles of the two O VI lines, which should be intrinsically identical except for peak intensity, differ from one another. This is because $\lambda 1031.9$ lies in a spectral region free of overlying $\mathrm{H}_{2}$ or interstellar absorption lines, while $\lambda 1037.6$ lies in a relatively complex region involving both $\mathrm{H}_{2}$ and interstellar (especially C II and $\mathrm{C} \mathrm{II*)} \mathrm{absorption,} \mathrm{at} \mathrm{both} \mathrm{galactic} \mathrm{and} \mathrm{LMC} \mathrm{veloci-}$ ties. The $\lambda 1031.9$ line, being less severely attenuated, is a better representation of the intrinsic line profile. Thirdly, comparing the C III $\lambda 977.0$ and O VI $\lambda 1031.9$ profiles, the absorption dips align at $\sim 260 \mathrm{~km} \mathrm{~s}^{-1}$. This is close to the expected mean redshift of the LMC, and we thus attribute these dips to C III and O VI absorption by diffuse LMC gas along the line of sight to the SNR. The absorption must be present in O VI $\lambda 1037.6$ as well, but is largely masked by other overlying absorptions. Any similar absorption from the Milky Way halo along this line of sight would be shortward of the blue wing of these lines. It is clear that the C III line is affected by overlying $\mathrm{H}_{2}$ absorption, especially on the red wing, where a plateau of emission is seen out to the full extent of the O VI velocity profile, but at a reduced level compared with O VI.

The absence of N III $\lambda 989.8$ in the observed spectrum is interesting. This line traces the same ionization state and gas temperature as C III $\lambda$ 977.0. Thus, in the recombination zones behind radiative shocks, both lines are emitted from the same region. The relative intensity of the N III line to the C III line is therefore a direct measure of the relative abundances of $\mathrm{N}$ and $\mathrm{C}$, and is less dependent on shock velocity or pre-shock density.

To obtain a measure of the $\mathrm{N}$ III to $\mathrm{C}$ III line ratio in N49, we ran shock models using an updated version of the code described by Raymond (1979). These models were run with the LMC abundances reported by Dufour, Shields, \& Talbot (1982), with $\mathrm{C}=7.90$ and $\mathrm{N}=6.97$ (on a logarithmic scale with $\mathrm{H}=12$ ). For shock velocities between $200 \mathrm{~km} \mathrm{~s}^{-1}$ and $300 \mathrm{~km} \mathrm{~s}^{-1}$ and a pre-shock density of $30 \mathrm{~cm}^{-3}$ (cf. Vancura et al. 1992a), the N III $\lambda 989.8$ to C III $\lambda 977.0$ ratio is about 0.06 or less. (Using abundances from Vancura et al. 1992a and a power law grid of models yields an even lower expected ratio of $\sim 0.03$.)

The region of the spectrum around $990 \AA$ has several $\mathrm{H}_{2}$ lines (Figure 2b) and interstellar lines that could absorb $\mathrm{N}$ III line photons and make it effectively unobservable. To investigate this possibility we ran simulated FUSE spectra and then attenuated them by assumed overlying absorption. The C III and N III lines were modeled as gaussians with FWHM $\sim 270 \mathrm{~km} \mathrm{~s}^{-1}$. The peak C III flux was taken to be the observed value, and two cases for the N III peak were considered -0.1 and 0.5 times the C III peak intensity. We used two components for the absorbing gas, one at $0 \mathrm{~km} \mathrm{~s}^{-1}$ and the other at $250 \mathrm{~km} \mathrm{~s}^{-1}$, corresponding to Milky Way and LMC velocities, respectively. Each component had a kinetic temperature of $250 \mathrm{~K}$ and an $\mathrm{H}_{2}$ column of $10^{18} \mathrm{~cm}^{-2}$. We find that this simple model for the absorbing gas severely attenuates the N III line, completely absorbing N III in the weaker line assumption and nearly removing it in the stronger line assumption.

It is possible that other interstellar lines contribute towards blanketing the N III line. In particular, based on Si II lines at longer wavelengths (cf. Welty et al. 1999), we expect Si II $\lambda 990$ to be a significant absorber. Also, 
self-absorption by LMC gas, as seen in the other strong lines, should contribute to removing the line.

We note that similar attenuation must happen elsewhere in the spectrum as well. The same model used above for N III produces the attenuation we see in the O VI $\lambda 1037.6$ line at least qualitatively. Using this model to make a first order correction to the strength of the $1037.6 \AA$ line indicates that the intrinsic ratio of O VI $\lambda 1031.9 / \lambda 1037.6$ is $\sim 1.7$, somewhat lower than the optically thin value of 2.0. This indicates that some of the O VI emitting regions within the aperture are optically thick.

\subsection{Weak Lines}

One of the hallmarks of shock heated gas is the simultaneous presence of a broad range of ionization states of many ions. The expected emission from the hotter components is similar to that from coronal plasmas (cf. Feldman et al. 1997).

In Figure 4, we show several small spectral regions containing some of the fainter lines from N49 detected with FUSE. [Ne V] $\lambda \lambda 1137,1146, \mathrm{~S}$ VI $\lambda \lambda 933,944$, and Ne VI $\lambda \lambda 999,1006$, are from temperature regions of $200,000-$ 500,000 K (cf. Mazzotta et al. 1998), intermediate to $\mathrm{X}$-ray emitting gas and regions sampled by the cooler ions observed at longer wavelengths, and similar to O VI. However, the abundances of $\mathrm{Ne}$ and $\mathrm{S}$ are much lower than $\mathrm{O}$, attesting to the sensitivity of FUSE for observing faint, diffuse sources. Also shown in Figure 4 is a region near $1085 \AA$, showing a faint line with possible absorption blueward from line center as in the stronger lines. This is either the He II $\lambda 1084.5$, or the N II $\lambda 1084.0$ line with self-absorption at the LMC velocity. We also see evidence for a faint broad line near $1074 \AA$ which we identify with S IV $\lambda$ 1073.0. Another S IV line expected near $1063.5 \AA$ appears to be marginally present, but is expected to be attenuated by overlying $\mathrm{H}_{2}$ absorption. Although many of these lines have been detected at a marginal level in HUT spectra, none of these faint lines have been detected previously in an extragalactic SNR. With future detailed modeling, these lines will provide new constraints on the cooling plasma behind interstellar shock waves.

It is a pleasure to thank the hundreds of people at our collaborating universities and institutions who worked on the development phase of FUSE, and the many scientists and engineers who have checked out the instrument and are now operating it on orbit. This work is based on data obtained for the Guaranteed Time Team by the NASACNES-CSA FUSE mission operated by the Johns Hopkins University. Financial support to U. S. participants has been provided by NASA contract NAS5-32985.

\section{REFERENCES}

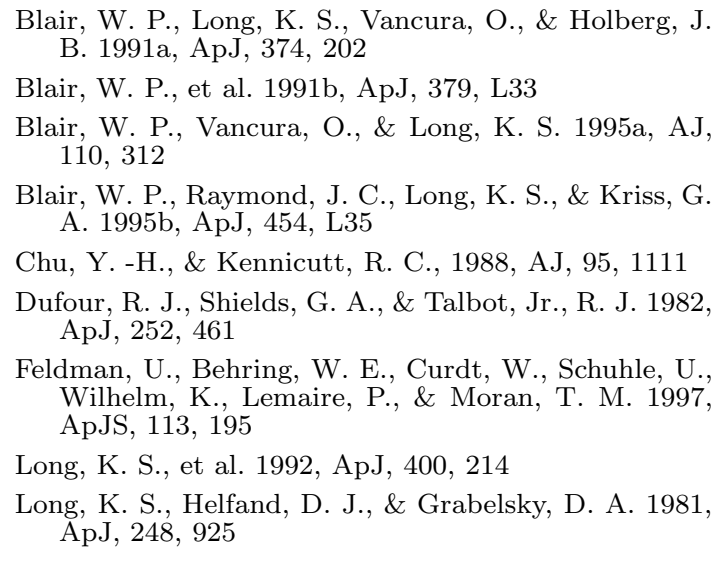

Mathewson, D. S., Ford, V. L., Dopita, M. A., Tuohy, I. R., Long, K. S., \& Helfand, D. J. 1983, ApJS, 51, 345

Mazzotta, P., Mazzitelli, G., Coalfrancesco, S., \& Vittorio, N. 1998, A\&AS, 133, 403

Moos, H. W., et al. 2000, ApJ, (this volume)

Raymond, J. C. 1979, ApJS, 39, 1

Sahnow, D., et al. 2000, ApJ, (this volume)

Shelton, R., et al. 2000, ApJ, in preparation

Shull, P. O. 1983, ApJ, 275, 611

Vancura, O., Blair, W.P., Long, K. S., \& Raymond, J. C. 1992a, ApJ, 394, 158

Vancura, O., et al. 1992b, ApJ, 401, 220

Vancura, O., Blair, W.P., Raymond, J.C., \& Holberg, J.B. 1993, ApJ, 417, 663

Welty, D. E., Frisch, P. C., Sonneborn, G., \& York, D. G. 1999, ApJ, 512, 636

\section{Figure Captions:}

Figure 1: Optical [O III] CCD image of N49 in the LMC, from Vancura et al. (1992a). Overlaid as a solid box is the position of the $30^{\prime \prime}$ aperture viewed by the guidance camera on FUSE. The offset dashed box shows the approximate position of the $\mathrm{SiC} 2$ channel, which is more uncertain in the vertical direction than in the horizontal direction. The $\mathrm{SiC} 1$ channel was not yet aligned at the time of this observation. North is up and east to the left, and the spatial scale can be derived from the aperture overlays.

Figure 2: Selected regions from the FUSE spectrum of N49 showing the strongest lines detected. a) the region from $1020-1045 \AA$ containing the O VI doublet, and b) the region from $970-995 \AA$ covering C III $\lambda 977.0$. Note the absence of any obvious N III $\lambda 991$ emission. Narrower lines arise from airglow emission lines and indicate the line widths expected from a filled aperture.

Figure 3: The three strongest SNR lines shown on a velocity scale. The middle line corresponds to O VI $\lambda 1031.9$ and is least affected by overlying absorption from $\mathrm{H}_{2}$ and interstellar lines. Shown on top is C III $\lambda 977.0$ and below is the other O VI line.

Figure 4: Some of the fainter lines detected in the FUSE spectra of N49. a) S VI $\lambda \lambda 933.4,944.5$; b) Ne VI $\lambda \lambda 999.2,1005.7$; c) $[\mathrm{Ne} \mathrm{V}] \lambda \lambda 1136.5,1145.6$, and d) a blend of He II and N II near $1085 \AA$. 
TABLE 1

OBSERVED LINE STRENGTHS

\begin{tabular}{cccc}
\hline \hline Ion & $\lambda$ & Flux & Channel \\
\hline C III & 977 & $1.9 \times 10^{-13}$ & SiC 2A \\
O VI & 1032 & $5.8 \times 10^{-13}$ & Combined \\
O VI & 1038 & $2.2 \times 10^{-13}$ & Combined \\
O VI & 1032 & $6.4 \times 10^{-13}$ & LiF 1A \\
O VI & 1038 & $2.2 \times 10^{-13}$ & LiF 1A \\
O VI & 1032 & $5.7 \times 10^{-13}$ & LiF 2B \\
O VI & 1038 & $2.1 \times 10^{-13}$ & LiF 2B \\
O VI & 1032 & $5.5 \times 10^{-13}$ & SiC 2B \\
O VI & 1038 & $2.3 \times 10^{-13}$ & SiC 2B \\
& & & \\
S VI & 933 & $1.1 \times 10^{-14}$ & SiC 2A \\
S VI & 945 & $1.2 \times 10^{-14}$ & SiC 2A \\
Ne VI & 1000 & $1.5 \times 10^{-14}$ & LiF 1A \\
Ne VI & 1006 & $1.2 \times 10^{-14}$ & LiF 1A \\
He II+N II & 1085 & $2.7 \times 10^{-14}$ & SiC 2B \\
[Ne V] & 1136 & $0.9 \times 10^{-14}$ & LiF 1B \\
[Ne V] & 1146 & $1.6 \times 10^{-14}$ & LiF 1B \\
\hline
\end{tabular}

Note.-These are observed values, uncorrected for reddening and absorption. The units are erg $\mathrm{s}^{-1} \mathrm{~cm}^{-2}$. 